\title{
Barriers to Advancement in Academic Medicine: the Perception Gap Between Majority Men and Other Faculty
}

\author{
Lori Brand Bateman, $P h D^{1,3}$ (D), Laura Heider, $M B A^{2}$, Selwyn M. Vickers, MD, FACS ${ }^{7}$, \\ William A. Anderson, $P h D^{7}$, Anthony C. Hood, $P h D^{4}$, Evelyn Jones, $M A^{7}$, \\ Corilyn Ott, $\mathrm{PhD}^{7,5}$, Sequoya Eady, $\mathrm{MPH}^{7}$, and Mona N. Fouad, $\mathrm{MD}, \mathrm{MPH}^{7}$
}

\begin{abstract}
'School of Medicine, The University of Alabama at Birmingham, Birmingham, AL, USA; ${ }^{2}$ Office for Diversity and Inclusion, Oschner Health, Baton Rouge, LA, USA; ${ }^{3}$ Division of Preventive Medicine, UAB School of Medicine, 171711 th Avenue South, Birmingham, AL, USA; ${ }^{4}$ Collat School of Business, The University of Alabama at Birmingham, Birmingham, AL, USA; ${ }^{5}$ School of Nursing, The University of Alabama at Birmingham, Birmingham, AL, USA.
\end{abstract}

BACKGROUND: According to the American Association of Medical Colleges, women comprise $26 \%$ of full professors and $19 \%$ of medical school department chairs. African American and Latino faculty comprise $4.6 \%$ of full professors and $6.9 \%$ of department chairs.

OBJECTIVE: Because of the lack of representation of women and racial/ethnic minority faculty at the highest levels of academic medicine, this study examines the perceptions of barriers to advancement by men and women academic medical school faculty of differing races and ethnicities to explore potential differences in perceptions by demographic group.

DESIGN: Semi-structured one-on-one interviews were conducted between July and September 2017.

Previous Presentations Bateman LB. Barriers to Advancement in Academic Medicine: A Qualitative Study of Differing Perceptions Based on Gender and Race/Ethnicity. Division of Preventive Medicine Grand Rounds, March 9, 2020, Birmingham, AL.

Bateman LB, Heider L, Fouad MN. Barriers and Facilitators to Advancement of Underrepresented Minorities and Women in Academic Medicine: Qualitative Findings from a Southeastern Academic Medical Center. UAB $14^{\text {th }}$ Annual Health Disparities Research Symposium, April 18, 2019, Birmingham, AL.

Bateman LB, Heider L, Fouad MN. Barriers and Facilitators to Advancement of Underrepresented Minorities and Women in Academic Medicine: Differing Perceptions Based on Race/Ethnicity and Sex. Southern Sociological Society Annual Meeting, April 10-13, 2019, Atlanta, GA.

Bateman LB, Heider L, Jones E, Fouad MN. Barriers and Facilitators to the Advancement of Women in Academic Medicine: Differing Perspectives Based on Gender and Race/Ethnicity. 2019 Region 5 American Medical Women's Association, February 15-16, 2019, Birmingham, AL.

Bateman LB, Heider L, Fouad MN. Barriers and Facilitators to the Advancement of Underrepresented Minorities in Academic Medicine: Ethical Implications for the Elimination of Health Disparities. Bioethics Issues in Minority Health and Health Disparities Research, sponsored by The Morehouse School of Medicine/Tuskegee University/University of Alabama at Birmingham Comprehensive Cancer Center Partnership, January 23-25, 2019, Opelika, AL.

Bateman LB, Heider L and Jones E. Diversity and Inclusion Programming in Academic Medicine: An Interactive Development Model. American Association of Medical Colleges Group on Diversity and Inclusion. June 1, 2018, New Orleans, LA.

Received June 16, 2020

Revised November 3, 2020

Accepted December 20, 2020

Published online January 27, 2021
PARTICIPANTS: In order to give all faculty a chance to participate, faculty of all ranks and specialties were recruited from one southeastern medical school to participate in the study.

APPROACH: Interviews were audio recorded, transcribed, and analyzed by 3 members of the research team using an inductive approach to thematic analysis. Participants were organized into 4 groups for analysis-underrepresented in medicine (URiM) women, majority women, URiM men, majority men.

KEY RESULTS: Sixty-four faculty consented to participate in the study (56.2\% women, 34.4\% URiM). Subthemes were grouped under three main themes: Perceptions of Barriers to Advancement of Women Faculty, Perceptions of Barriers to Advancement of African American and Latino Faculty, and Perceptions of the Institutional Climate for Diversity. Majority men tended to voice distinctly different perspectives than the other three demographic groups, with the most notable differences between majority men and URiM women. Majority men tended to suggest that the advancement of women and URiM faculty was acceptable or getting better, the lack of URiM faculty in leadership was due mainly to pipeline issues, and women choose not to advance to leadership positions.

CONCLUSION: We found that participant gender and race/ethnicity shaped perspectives of medical school faculty advancement in distinct ways.

KEY WORDS: academic advancement; academic medicine; underrepresented in medicine; women.

J Gen Intern Med 36(7):1937-43

DOI: $10.1007 / \mathrm{s} 11606-020-06515-5$

(c) Society of General Internal Medicine 2021

\section{INTRODUCTION}

According to the most recent data from the American Association of Medical Colleges (AAMC), women comprise $42.3 \%$ of medical school faculty, but only $26 \%$ of full professors, and $19 \%$ of medical school department chairs. Women are overrepresented in the lower ranks of academic medicine, making 
up 59\% of faculty filling instructor positions. ${ }^{1}$ Further, Black/ African American and Hispanic/Latino/of Spanish Descent faculty comprise only $6.9 \%$ of faculty overall, $4.6 \%$ of full professors, and $7.3 \%$ of department chairs. ${ }^{1}$ Given that $31.5 \%$ of the US population is African American and/or Latino, ${ }^{2}$ these numbers are alarmingly low.

Potential causes and implications have been explored at length in numerous studies, but concrete answers are scarce. ${ }^{3,4}$ Literature is replete with theories, including the paucity of mentoring, ${ }^{4,5}$ less robust negotiation skills, particularly with regard to women faculty, ${ }^{6}$ and fewer members of underrepresented groups at lower levels of the pipeline of academic medicine. ${ }^{7}$ Yet, even when accounting for these and other factors, a gap exists between majority men and both women and underrepresented minorities. ${ }^{3,4,7-9}$ While quantitative data is needed to track progress toward increasing the numbers of women and racial/ethnic minorities who are underrepresented in medicine (URiM), qualitative methods offer insight into lived experiences and allow for open-ended feedback. This combination is crucial to understanding the biases, institutional norms, unspoken assumptions, and systemic racism that reinforce systems of implicit (or unintentional) inequality. Understanding and addressing these issues are taking on increasing importance in the wake of numerous deaths of African Americans in police custody and the ensuing protests that have spread across the USA. Although racism within health care as an institution has been documented for many years, ${ }^{10-12}$ eliminating it has taken on heightened importance in light of recent calls by medical and health organizations to address it as an urgent and critical matter. ${ }^{13}$

It is not surprising that demographic groups have different perspectives. Previous qualitative studies have examined academic advancement from the perspectives of women and racial/ ethnic minorities, but very few have included the perspective of majority males. ${ }^{14-17}$ When one group, in this case white males, hold outsized power and influence, we risk basing recommendations for progress on incomplete or inaccurate data if we do not understand the degree to which majority males see their overrepresentation in positions of power as an issue. We further need to understand the factors to which they attribute their relative over-representation, for example, personal (e.g., work ethic, sacrifice), interpersonal (e.g., networking, mentoring), or institutional (e.g., leaky leadership pipelines, institutional policies, organizational culture), and what role they feel they should or should not play in addressing their relative over-representation.

Because of the lack of representation of women and racial/ethnic minority faculty at the highest levels of academic medicine, this study examines the perceptions of barriers to advancement by men and women academic medical school faculty of differing races and ethnicities. Complementing the extant research which primarily examines the perspectives of URiM and women faculty, the purpose of the current study is to address perceptions of barriers to academic advancement among faculty who are both under- and over-represented in academic medicine.

\section{METHODS}

Hurtado et al. ${ }^{18}$ suggest that factors unique to each institution go into creating its "institutional climate." These include the historical legacy of inclusion/exclusion (resistance to desegregation, mission, policies), structural diversity (numbers of diverse students and faculty), psychological climate (perceptions of racial/ethnic tension, perceptions of discrimination and attitudes of reduction of prejudice), and the behavioral dimension (social interaction across racial/ethnic groups, campus involvement and diversity, and classroom diversity). In order for programs to be effective in eliminating disparities in faculty advancement, we posit that the institutional climate must be examined through the lens of both over- and underrepresented faculty members.

This study was conducted at a single medical school in the Southeast employing 1300 faculty and the study protocol was approved by the Institutional Review Board of the university (see Table 1 for faculty demographics by rank). Because we wanted to give all faculty (regardless of rank or demographic group) the opportunity to participate, a recruitment email was sent in July 2017 to the School of Medicine faculty listserv, inviting all faculty members, both clinical and research, to participate in a $1 \mathrm{~h}$ or less, confidential face-to-face interview examining the institutional climate for diversity and perceived barriers and facilitators to advancement of women and URiM medical school faculty. Informed consent was obtained from all participants and a $\$ 50$ incentive was offered for participation. Sixty-four faculty consented to participate (56.2\% women, $34.4 \%$ URiM) (see Table 2 for participant demographics by rank).

Six interviewers conducted interviews between July and September 2017. When possible, interviewers were matched with participants with whom they were race and gender concordant, to make participants as comfortable as possible and minimize perceived power differentials between interviewer and interviewee. The interview guide was based on the Institutional Climate for Diversity, ${ }^{18}$ previous studies reported in the literature, and analysis of comments from a recent AAMC-developed Diversity Engagement Survey conducted at the institution (see Appendix 1 for interview guide). In order to maintain a conversational style, however, new themes brought up by participants were pursued. Advancement was defined as moving up in academic rank (from assistant to associate to full professor) or moving into leadership positions within the institution.

Interviews were audio recorded, transcribed, and stored electronically on a password-protected university storage site. Identifying information was removed from the transcripts and participants were assigned study numbers. Coding and analysis were overseen by the principal investigator. In order to explore differences in perspectives, participants were organized into 4 groups - URiM women, majority women, URiM men, and majority men. The AAMC's definition of URiM was used to classify participants. ${ }^{20}$ URiM participants included Black/African American faculty and Hispanic/Latino/of Spanish Descent faculty; there were no participants in other 
Table 1 School of Medicine Faculty Demographics by Rank $(\%)^{19}$

\begin{tabular}{lllll}
\hline \hline & Women & Men & Majority (White, Asian) & URiM (African American/Latino) \\
\hline All & 36.20 & 63.80 & 91.46 & 7.98 \\
Instructor/assistant professor & 44.50 & 55.50 & 89.97 & 9.07 \\
Associate professor & 44.50 & 63.92 & 91.76 & 8.24 \\
Full professor & 25.11 & 74.89 & 93.24 & 6.33 \\
\hline
\end{tabular}

underrepresented groups. Asian faculty were considered to be majority in keeping with the AAMC definition.

Members of the research team met regularly to review transcripts and determine if data saturation had been reached for each demographic group. At one point, another recruitment email was sent to URiM faculty as more interviews were needed. Data were analyzed inductively and guided by thematic analysis, ${ }^{21}$ and Nvivo 11 was utilized to organize the data. Transcribed data was read by two coders (LB and LH) to identify underlying concepts and to develop an initial coding structure. Data was coded line-by-line, and these initial codes were used to start revealing potential thematic categories. Memos were written by two coders throughout the coding process to continuously compare data and make connections between data. This allowed the authors to explore emerging themes while building linkages between concepts. ${ }^{22}$ Next, through focused coding, the two coders arranged concepts into codes that were more "directive, selective, and conceptu$\mathrm{al}^{1,22}$ than in the initial coding. These codes classified the data according to emerging themes. At this point, a third coder (CO) was invited to join the project from outside the department to check inherent bias that might exist within the research team. The three coders (LB, LH, CO) independently coded transcripts (each transcript was coded by differing combinations of two of the three coders) to enhance the trustworthiness of the data based on the emergent coding structure. They met regularly to discuss where coding matched and was divergent, and to reconcile differences. Throughout the process, codes were deleted, trimmed, renamed, and elevated into themes.

\section{RESULTS}

Themes were identified in three areas: Perceived Barriers to Advancement of Women Faculty, Perceived Barriers to Advancement of African American and Latino Faculty, and the Institutional Climate for Diversity (for a list of themes and subthemes, see Table 3; for representative quotes for each theme, see Appendix 2).

\section{Perceived Barriers to Advancement of Women Faculty}

Women's Advancement Getting Better. Majority men painted a more optimistic picture of women's advancement than other groups, with most stating that women's advancement was good or getting better. For example, one majority man stated, "I'm also on our Promotions Committee, and I don't see that there's any issues there. . . Gender-blind, I would agree with that." However, only one URiM woman and a few URiM men and majority women agreed that the climate for advancement was acceptable or improving.

Majority men also stated to a greater extent than other groups that the advancement of women in academic medicine would take place naturally, over time, based on the fact that men and women are now entering the medical profession at similar rates. One majority man said, "I think those kinds of differences will gradually improve over time. It may not get to completely equal (sic), just because there seems to be some attrition of women who decide not to work full-time, when they get to the upper career levels. ..."

Women Choose Family over Advancement. Majority men were likely to believe that women medical school faculty choose to pursue family over advancement, voluntarily eliminating themselves from leadership. For example, "My sense is that working mothers, even on a professional level, may have . . . bias against advancement, promotion, the responsibilities in promotion in rank." In contrast, only one URiM man mentioned this and only a few women suggested that women chose family over career advancement.

Working Mothers Have Difficulty Meeting the Demands of Advancement. All demographic groups shared the perspective that working mothers had difficulty fulfilling the demands

Table 2 Participants by Rank, Gender, and Race/Ethnicity

\begin{tabular}{|c|c|c|c|c|c|c|}
\hline & $\begin{array}{l}\text { URiM women } \\
N(\%)\end{array}$ & $\begin{array}{l}\text { Majority women } \\
N(\%)\end{array}$ & $\begin{array}{l}\text { URiM men } \\
N(\%)\end{array}$ & $\begin{array}{l}\text { Majority men } \\
N(\%)\end{array}$ & $\begin{array}{l}\text { Decline to identify/other } \\
\text { race women } \\
N(\%)\end{array}$ & $\begin{array}{l}\text { Total } \\
N(\%)\end{array}$ \\
\hline \multirow{5}{*}{$\begin{array}{l}\text { Instructor/assistant professor } \\
\text { Associate professor } \\
\text { Full professor } \\
\text { Decline to identify rank } \\
\text { Total }\end{array}$} & $7(11.0)$ & $9(14.1)$ & $3(4.7)$ & $6(9.4)$ & \multirow[t]{5}{*}{$1(1.6)$} & \multirow{5}{*}{$\begin{array}{l}26(40.1) \\
14(21.9) \\
23(35.9) \\
1(1.6) \\
64(100)\end{array}$} \\
\hline & $4(6.3)$ & $6(9.4)$ & 1 (1.6) & $3(4.7)$ & & \\
\hline & $4(6.3)$ & $4(6.3)$ & $3(4.7)$ & $12(18.8)$ & & \\
\hline & & & & & & \\
\hline & $15(23.4)$ & $19(29 / 7)$ & 7 (10.9) & $21(32.8)$ & & \\
\hline
\end{tabular}


Table 3 Table of Themes and Subthemes

\begin{tabular}{ll}
\hline \hline Theme & Subtheme \\
\hline Perceptions of Barriers to Advancement of Women Faculty & Women's advancement getting better \\
& Women choose family over advancement \\
& Working mothers have difficulty meeting the demands of advancement \\
& Bias impacts women's advancement \\
Perceptions of Barriers to Advancement of African & URiM advancement getting better \\
American and Latino Faculty & URiMs don't advance due to pipeline issues \\
& URiMs don't advance because of bias \\
Perceptions of the Institutional Climate for Diversity & URiMs must work harder to advance \\
& Equal opportunity to advance \\
& Treated with respect \\
& Feeling observing racial tension \\
\hline
\end{tabular}

required for advancement because of added responsibilities of parenting, with one-third of participants mentioning this. However, male participants more often suggested that women were responsible for this difficulty, whereas women tended to see it as a problem with the institution, which does not value family commitments, or with society, in which women are assumed to be the primary caregivers for their families. For example, one majority man stated, "I'm not sure that it reflects a bias against women as much as a failure to adapt to competing demands...more women than men also maintain responsibility for children and child care." Juxtaposed to this view was the perspective of women faculty, who tended to place the difficulty of advancing at the feet of the institution and/or society. One majority woman said, “. . . Well, look. John got all these papers written during this time. Why do I need to give you academic time to write papers? Well, John's also not cleaning house, cooking dinner, folding laundry, cleaning up after the sick dog or the sick kid."

Bias Impacts Women's Advancement. In addition to familyrelated issues, all groups mentioned that bias affected women's advancement to some extent, with URiM women suggesting this most often. Divergent views based on gender were seen in three themes related to bias. First, over half of all women brought up the idea of a "good old boys club" or lack of gender representation as a reason why women have difficulty advancing and reaching leadership positions. Less than a quarter of men mentioned this as a reason. Second, more than half of the women respondents mentioned the importance of mentoring and its lack of availability as a key factor; only two men discussed this. Finally, almost half of women discussed the idea that women have to work harder to achieve the same status of men in academic medicine, while few men mentioned this idea.

\section{Perceived Barriers to Advancement of African American and Latino Faculty}

URiM Advancement Getting Better. Just as majority men saw advancement for women as either good or getting better, about half of them also suggested the same was true for URiM faculty. Only a few members of the other groups mentioned this, however. The same three men in key leadership positions were mentioned by multiple male participants as evidence that URiMs no longer have difficulty advancing.

URiMs Don't Advance due to Pipeline Issues. Perceptions of what factors prevented URiM faculty from advancing varied by demographic group. About half of all participants suggested pipeline issues play a role in URiM advancement. But majority men brought pipeline issues up to a greater extent, with most suggesting pipeline issues explain part or all of the disparity in advancement between majority and URiM faculty. A majority male suggested that issues related to the pipeline were solely responsible for the lack of URiM advancement: "There just aren't enough qualified minorities for these jobs - just not. . . There aren't qualified people out there ... totally a pipeline problem."

URiMs Don't Advance Because of Bias. All groups discussed bias as a reason that kept URiMs from advancing and reaching the highest levels in medicine, with at least half of URiMs bringing this up. At least one-third of majority men also mentioned this. One female URiM faculty member stated, "You can't really have an opinion if you're a woman of color. You could be there, but really not be controversial, look controversial, or look too ethnic. I think that yes, people like diversity, but I think there's a certain cookie-cutter type of ethnicity that they like."

URiMs Must Work Harder to Advance. A final emergent theme is that URiM faculty must work harder than their majority counterparts to attain the same level of advancement. Roughly one-third of URiM faculty suggested that one reason for this is that URiM faculty are asked to fulfill minority representation on committees or mentoring, which places an undue burden on faculty who may already be subject to bias. Only a few non-URiM participants mentioned this as a factor. For example, an URiM woman called the extra work a "tax": "That goes back to . . . sometimes being on things that I'm like, why I am here? I'm clearly helping somebody address a need, right, but it's not really fulfilling my need. . . There's that added tax or burden that faculty from underrepresented groups have that can impede success." 


\section{Perceptions of the Institutional Climate for Diversity}

Equal Opportunity to Advance. When asked if individuals from different backgrounds had an equal opportunity to succeed in the School of Medicine, roughly three-quarters of majority men said "yes," while approximately three-quarters of URiM women said, "no." In fact, only one URiM woman said, "yes" with no caveats. For example, a majority man responded, "Yes ... This is one of the most diverse environments ... you could work in, really." URiM women, however, painted a different picture in their responses as the following indicates, "No. I'm a clear illustration of that. I did it despite tremendous obstacles." Most URiM men and majority women said that individuals did not have equal opportunities or that they were unsure whether they did or not.

Treated with Respect. Further, we found that whether or not participants felt that they were treated with respect at work varied by demographics, with majority men feeling the most respected and URiM women feeling the least so. Almost all majority men stated that they felt respected when at work. One representative response from a majority male: "Definitely. Probably more respect than I ever had." In contrast, most URiM women said that they did not feel respected or that they were unsure, such as seen in this response, "I think that for most people who are minorities... Once you get to a certain point, it's like... 'When am I actually gonna get rewarded for what I'm doing?'... You're just doing all this stuff, and someone else is sometimes getting the credit for it." Only a few URiM women said that they felt respected with no contingencies.

Most URiM men said that they felt respected, but provided personal stories that illustrated caveats or qualifiers to their experience of respect. For example, one participant said others joked about his Hispanic last name. Majority women also tended to state they felt respected, with only one responding that she did not feel respected and a few conveying mixed responses.

Feeling and/or Observing Racial Tension. Finally, we also found that whether or not one observed racial tension was based on demographics, with URiM women diverging from the other groups. Only a few majority men and women and URiM men said they observed racial tension at work, although many were quick to point out that it may exist but they did not experience it personally. Most URiM women, however, said they did observe racial tension. For example, one URiM woman stated, "I feel that - so there's a lot of things that aren't said... because any time you bring racial issues up, there's always gonna be some type of controversy."

\section{DISCUSSION}

These findings suggest that perceptions of academic advancement vary by demographic group membership, and that numerous systemic and attitudinal barriers to the advancement of women and underrepresented minorities still play an important role in the career development of those traditionally marginalized. However, this study moves beyond these insights by exploring the gap between the perceptions of majority men and other demographic groups. In our study, URiM men, URiM women, and majority women had differing views from majority males in their perceptions of both the existence and nature of barriers to the advancement of women and underrepresented minorities.

This study affirmed the existence of bias against motherhood, as many majority males seemed to place some of the responsibility for women's lack of advancement on women who were mothers who, they argued, were likely to choose family over career. Numerous studies across fields have noted that women pay a "motherhood penalty" for having children, regardless of experience or level of education, in which they are perceived as being less committed to their careers, less likely to be suggested for promotion, and more likely to receive a lower starting wage than childless women. ${ }^{23}$ In the USA, researchers have identified a $7 \%$ wage penalty per child, only one-third of which can be attributed to the loss of work experience. ${ }^{24}$ By contrast, men receive a "bonus" or "premium" for becoming fathers. ${ }^{23}$

The current study also indicates that majority men may be unaware or insensitive to gender issues such as male-oriented networking and mentoring opportunities and the need on the part of women faculty to overcome gendered stereotypes. Believing that issues influencing the advancement of women will naturally resolve themselves over time allows men to absolve themselves of personal responsibility and leaves them unwilling or incapable of proactively leading or supporting efforts to address such issues. Leadership must become aware of the perspective of women faculty members with regard to concerns about their advancement in order to assist in alleviating these barriers.

In terms of the advancement of members of racially underrepresented groups, majority men and URiM women tended to diverge in their perceptions of URiM advancement. Although at least half of majority men suggested that advancement for URiM faculty was acceptable and getting better, no URiM women posited this notion. Consistent with much of the extant literature surrounding the paucity of members of underrepresented minorities at the highest reaches of academic medicine, ${ }^{4,7}$ participants from all groups suggested pipeline issues play a role. But, most majority men suggested that pipeline issues explain part or all of the disparity in advancement between majority and URiM faculty, while women and underrepresented minority faculty members tended to suggest that other factors, such as bias or structural barriers, explained a great deal of the disparity.

While studies on the lack of diversity in academic medicine support the idea of pipeline issues as an important factor, they also point to institutional culture, re-examining hiring and promotion criteria for bias, and aggressive outreach as equally crucial to the quest for race-equitable representation. ${ }^{25,} 26$ Other factors which researchers have found to contribute to the lack of 
advancement of URiMs include inadequate development opportunities, ${ }^{26}$ inadequate mentoring opportunities, ${ }^{15,}{ }^{16}$ isolation, ${ }^{15}$, 27,28 and a sense of being overburdened with additional work due to their minority status. ${ }^{28,}{ }^{29}$ Indeed, the URiM faculty in this study often mentioned the necessity of working harder than their majority counterparts to advance to the same level, and all groups mentioned racial bias as a reason for lack of advancement. Racial bias is consistently mentioned throughout research literature as a key obstacle to the advancement of racial/ethnic minorities. ${ }^{28-30}$ The characterization of lack of minority representation in academic medicine as a pipeline issue is, therefore, a substantial oversimplification which absolves (predominantly White) leadership of responsibility and makes it unlikely that they will address systemic institutional factors. Because majority males as a group tended to feel respected by their institution, it is possible that they are less likely to feel that the institution is burdened by systemic bias in need of rectifying.

Racism in health care, including among leaders in academic medical centers, is systemic and multidimensional. However, much of the race-critical literature does not name or examine the White males who control medical institutions and who operate out of a White racial framing. ${ }^{12}$ White racial framing includes racial bias, racial ideologies, narratives, emotions, and inclinations to discriminate. It is important that these leaders are compelled to listen to underrepresented voices about their experiences with institutionalized racism as well as their solutions. ${ }^{12}$ This study is an important step in that direction in that it highlights the divergence of perspectives based on lived experiences of structural bias. The contrast between these perspectives draws attention to the limitations of the majority perspective in perceiving structural barriers.

\section{Limitations}

The small sample size of URiM men may have limited our ability to understand the perspective of that group. Although, we combined URiM groups for analysis purposes, we acknowledge there may be differences in perspectives between URiM groups (i.e., Latinos vs. Blacks). Further, because our study is based on the AAMC definition of URiM, Asians were included in the majority group. We realize that this may have masked the unique perspectives that this group had in terms of advancement. Also, selection bias is likely in that participants volunteered to be in the study by responding to an email sent to all faculty in the School of Medicine, and respondents may have been more extreme on either side of the spectrum.

Another limitation relates to the race and gender concordance of the interviewer-interviewee or lack thereof in terms of reflexivity. ${ }^{31}$ To minimize potential bias that can occur in both situations, we assembled a team of diverse researchers to serve in a peer consultation role to check biases in interviewing, coding, and analysis. ${ }^{31}$ Finally, as with all qualitative studies, only a fraction of faculty in the institution were represented, and therefore, our conclusions cannot be seen as generalizable.

\section{CONCLUSION}

We uncover perceptions of how and why some and not others advance to positions of leadership and how these perspectives differ across lines of race/ethnicity and gender. In so doing, we seek to inform current efforts to address the unconscious biases and systemic structural barriers that promote and sustain inequities in leadership in academic medicine. This study suggests that a possible reason for the underrepresentation of women and underrepresented minorities in senior and leadership positions in academic medicine is that majority males may not perceive systemic bias against women or racial/ethnic minorities. This may render majority males, including those in leadership, less likely to work to eliminate systemic and historical barriers, leaving women and underrepresented minorities responsible for removing obstacles to their advancement that they did not create.

Given that the perception and acknowledgement of barriers is a necessary pre-requisite to active engagement in work to combat these barriers, diversity and inclusion initiatives which endeavor to broaden the perspectives of majority males might prove efficacious over the long run. Training on implicit/unconscious bias, town halls, and panel discussions at which women and underrepresented minority faculty and staff share their experiences, and institutional accountability for equity in recruitment and promotion practices could all play a role in this enterprise. Moreover, the intentional creation of institutional goals related to the recruitment and retention of women and URiM faculty might function as the necessary impetus to encourage local leadership, such as department chairs, to assume personal responsibility for altering internal practices. This work may be necessary to narrow the gap in perception between different demographic groups before diversity and inclusion efforts can achieve maximum efficacy.

Supplementary Information The online version contains supplementary material available at https://doi.org/10.1007/s11606-02006385- $x$.

Corresponding Author: Lori Brand Bateman, PhD; Division of Preventive Medicine, UAB School of Medicine, 1717 11th Avenue South, Birmingham, AL, USA (e-mail: loribateman@uabmc.edu).

Funding This project was supported through an institutional grant from the University of Alabama at Birmingham Office of Diversity and Inclusion.

\section{Compliance with Ethical Standards:}

Conflict of Interest: The authors declare that they do not have a conflict of interest.

Ethical Approval: The study was approved by the Institutional Review Board for Human Use at The University of Alabama at Birmingham (UAB), June 21, 2017, Protocol \# 170524006.

Disclaimers: None 


\section{REFERENCES}

1. AAMC. 2019 U.S. Medical School Faculty. 2020; https://www.aamc.org/ data-reports/faculty-institutions/interactive-data/2019-us-medicalschool-faculty. Accessed September 10, 2020.

2. Bureau UC. 2010 Decennial Census. 2010; https://www.census.gov/ programs-surveys/decennial-census/decade.2010.html. Accessed October 24, 2018.

3. Butkus R, Serchen J, Moyer DV, Bornstein SS, Hingle ST. Achieving Gender Equity in Physician Compensation and Career Advancement: A Position Paper of the American College of Physicians. Ann Intl Med. 2018.

4. Kaplan SE, Raj A, Carr PL, Terrin N, Breeze JL, Freund KM. Race/ Ethnicity and Success in Academic Medicine: Findings From a Longitudinal Multi-Institutional Study. Acad Med. 2018;93(4):616-622.

5. Robinson JD, Cannon DL. Mentoring in the academic medical setting: The gender gap. J Clin Psychol Med Settings. 2005; 12(3):265-270.

6. Freund KM, Raj A, Kaplan SE, et al. Inequities in academic compensation by gender: A follow-up to the National Faculty Survey cohort study. Acad Med: J Assoc Am Med Coll. 2016;91(8):1068.

7. Yu PT, Parsa PV, Hassanein O, Rogers SO, Chang DC. Minorities struggle to advance in academic medicine: A 12-y review of diversity at the highest levels of America's teaching institutions. J Surg Res. 2013;182(2):212-218.

8. Su X, Johnson J, Bozeman B. Gender diversity strategy in academic departments: exploring organizational determinants. Higher Educ. 2015;69(5):839-858.

9. Laurent L, Sosenko T, Zamfirova I, Hartwig C. Exploring equality in leadership roles. Phys Leadersh J. 2017;4(3):36-40.

10. Smedley BD, Stith AY, Nelson AR. Unequal Treatment: Confronting Racial and Ethinic Disparities in Health Care. Washington, D.C.: The National Academy Press; 2002.

11. King G. Institutional racism and the medical/health complex: a conceptual analysis. Ethn Dis. 1996;6(1-2):30-46.

12. Feagin J, Bennefield Z. Systemic racism and US health care. Social Sci Med. 2014;103:7-14.

13. Serchen J, Doherty R, Atiq O, Hilden D. Racism and health in the United States: A policy statement from the American College of Physicians. Ann Intl Med. 2020.

14. Isaac C, Griffin L, Carnes M. A qualitative study of faculty members' views of women chairs. J Women's Health. 2010;19(3):533-546.

15. Pololi L, Cooper LA, Carr P. Race, disadvantage and faculty experiences in academic medicine. J Gen Intern Med. 2010;25(12):1363-1369.

16. Mahoney MR, Wilson E, Odom KL, Flowers L, Adler SR. Minority faculty voices on diversity in academic medicine: perspectives from one school. Acad Med. 2008;83(8):781-786.
17. Pololi LH, Jones SJ. Women faculty: An analysis of their experiences in academic medicine and their coping strategies. Gender Med. 2010;7(5):438-450.

18. Hurtado S, Milem J, Clayton-Pedersen A, Allen W. Enacting Diverse Learning Environments: Improving the Climate for Racial/Ethnic Diversity in Higher Education. ASHE-ERIC Higher Education Report, Vol. 26, No. 8. ERIC; 1999

19. University of Alabama at Birmingham, School of Medicine, Office for Diversity and Inclusion, Diversity Dashboard. https://www.uab.edu/ medicine/diversity/diversity-toolkit/dashboard. Accessed 26 March 2020.

20. AAMC. Underrepresented in Medicine Definition. 2020; https://www. aamc.org/what-we-do/mission-areas/diversity-inclusion/underrepresented-in-medicine. Accessed 10 Sept 2020.

21. Braun V, Clarke V, Hayfield N, Terry G. Thematic analysis. Handb Res Methods Health Soc Sci. 2019;843-860.

22. Charmaz K. Constructing Grounded Theory: A Practical Guide Through Quantitative Analysis. Los Angeles: Sage; 2006.

23. Glauber R. Trends in the motherhood wage penalty and fatherhood wage premium for low, middle, and high earners. Demography. 2018;55(5): 1663-1680.

24. Misra J, Strader E. Gender pay equity in advanced countries: The role of parenthood and policies. J Int Aff. 2013;27-41.

25. Grumbach K, Mendoza R. Disparities in human resources: addressing the lack of diversity in the health professions. Health Aff. 2008;27(2):413422 .

26. Chen JA, Rodriguez JE, Campbell KM. Examining Diversity Offices in Medical Education. J Best Pract Health Prof Divers. 2018;11(1):43-50.

27. Pololi LH, Evans AT, Gibbs BK, Krupat E, Brennan RT, Civian JT. The experience of minority faculty who are underrepresented in medicine, at 26 representative U.S. medical schools. Acad Med. 2013;88(9):13081314.

28. Nivet MA, Taylor VS, Butts GC, et al. Diversity in academic medicine no. 1 case for minority faculty development today. Mt Sinai J Med. 2008;75(6):491-498.

29. Cohen JJ, Gabriel BA, Terrell C. The case for diversity in the health care workforce. Health Aff. 2002;21(5):90-102.

30. Peterson NB, Friedman RH, Ash AS, Franco S, Carr PL. Faculty selfreported experience with racial and ethnic discrimination in academic medicine. J Gen Intern Med. 2004;19(3):259-265.

31. Berger R. Now I see it, now I don't: Researcher's position and reflexivity in qualitative research. Gual Res. 2015;15(2):219-234.

Publisher's Note: Springer Nature remains neutral with regard to jurisdictional claims in published maps and institutional affiliations. 